\title{
The LeFE algorithm: embracing the complexity of gene expression in the interpretation of microarray data Gabriel S Eichler ${ }^{* \dagger}$, Mark Reimers**, David Kane ${ }^{*}$ and John N Weinstein*
}

\begin{abstract}
Addresses: *Genomics and Bioinformatics Groups, Laboratory of Molecular Pharmacology, Center for Cancer Research, National Cancer Institute, National Institutes of Health, Bethesda, Maryland 20892, USA. 'Bioinformatics Program, Boston University, Cummington St, Boston, Massachusetts 02215, USA. *Virginia Commonwealth University, Biostatistics Department, E Marshall St, Richmond, Virginia 23284,
\end{abstract} USA. §SRA International, Fair Lakes Court, Fairfax, Virginia 22033, USA.

Correspondence: John N Weinstein. Email: weinstein@dtpax2.ncifcrf.gov

Published: 10 September 2007

Genome Biology 2007, 8:RI87 (doi:10.1 |86/gb-2007-8-9-r|87)

The electronic version of this article is the complete one and can be found online at http://genomebiology.com/2007/8/9/RI87
Received: 15 February 2007

Revised: 29 June 2007

Accepted: 10 September 2007

(c) 2007 Eichler et al.; licensee BioMed Central Ltd.

This is an open access article distributed under the terms of the Creative Commons Attribution License (http://creativecommons.org/licenses/by/2.0), which permits unrestricted use, distribution, and reproduction in any medium, provided the original work is properly cited.

\begin{abstract}
Interpretation of microarray data remains a challenge, and most methods fail to consider the complex, nonlinear regulation of gene expression. To address that limitation, we introduce Learner of Functional Enrichment (LeFE), a statistical/machine learning algorithm based on Random Forest, and demonstrate it on several diverse datasets: smoker/never smoker, breast cancer classification, and cancer drug sensitivity. We also compare it with previously published algorithms, including Gene Set Enrichment Analysis. LeFE regularly identifies statistically significant functional themes consistent with known biology.
\end{abstract}

\section{Background}

Data from microarrays and other high-throughput molecular profiling platforms are clearly revolutionizing biological and biomedical research. However, interpretation of the data remains a challenge to the field and a bottleneck that limits formulation and exploration of new hypotheses. In particular, it has been a challenge to link gene expression profiles to functional phenotypic signatures such as those of disease or response to therapy. A number of partial bioinformatic solutions have been proposed. The most mature and promising such algorithms have analyzed the data from the perspective of categories of related genes, such as those defined by the Gene Ontology (GO) or by the Kyoto Encyclopedia of Genes and Genomes [1]. Gene categories group genes into nonexclusive sets of biologically related genes by linking genes of common function, pathway, or physical location within the cell.

Gene categories introduce an independent representation of the underlying biology into the analysis of complex datasets and therefore serve to guide the algorithms toward conclusions congruent with conventional knowledge of biological systems. Algorithms that take such an approach have often demonstrated a higher level of functional interpretation than did earlier, single-gene statistical analyses. However, most gene category based methods still perform the analysis on a gene-by-gene, univariate basis, failing to capture complex nonlinear relationships that may exist among the category's genes. If, for example, upregulation of gene A influenced a drug sensitivity signature only if gene B in the category were downregulated and gene $\mathrm{C}$ upregulated, then that relationship would be missed. Here, we introduce a novel gene category based approach, the Learner of Functional Enrichment (LeFE) algorithm, to the interpretation of microarray (and similar) data. LeFE captures that type of complex, systemsoriented information for prediction of functional signatures.

The input to LeFE consists of the following components: signature vector, microarray (or analogous) data, and a 
predefined set of categories and the genes within them. The 'signature vector' describes the biological behavior, process, or state to be predicted for each experimental sample. The signature vector either classifies samples (for example, as normal or diseased) or assigns each sample a continuous value (for example, relative drug sensitivity). That is, the signature can be nominal or continuous. A discrete signature vector is handled as though it were continuous.

The goal of LeFE or any other gene category based algorithm is to determine which categories (for instance, molecular subsystems) are most strongly associated with the biological states described by the signature vector. Toward that end, most previously published methods, for example Gene Set Enrichment Analysis (GSEA) [2], assign each gene category a score based on nonparametric statistics, $t$-statistics, or correlations that reflect the relationships between individual genes and the signature vector. The gene categories most enriched with those strong single-gene associations are said to be related to the signature. The degree of enrichment is usually represented by a $P$ value or false discovery rate using, for example, a Fisher's exact test $[3,4]$, a weighted Kolmogorov Smirnov test [2], or comparison with a $\chi^{2}$ [5], binomial [6], or hypergeometric [7] distribution. Although those approaches have proved useful, they neglect the fact that gene products generally function in complicated pathways or complexes whose expression patterns may not be reflected in the summation of univariate associations between single genes and the biological activity [8-11].

To address that shortcoming, LeFE uses a machine learning algorithm to model the genome's complex regulatory mechanisms, determining for each category whether its genes are more important as predictors (variables) than are a set of randomly sampled negative control genes. Although any of several different machine learning algorithms could be used in LeFE, we chose the Random Forest algorithm [12] because it has features (discussed below) that make it particularly apt for this application. The power of Random Forest has been successfully demonstrated in numerous bioinformatic and chemoinformatic applications [13-16]. As per the 'no free lunch' dictum [17], no single machine learning algorithm can be optimal for all datasets and applications, but Random Forest appears to be an appropriate choice as an engine for LeFE.

The Random Forest algorithm builds an ensemble of decision trees using the Classification and Regression Tree (CART) method [18]. Random Forest is therefore included among the general class of 'ensemble learning' algorithms. The algorithm injects diversity into the tree creation process by building each tree on an independently bootstrapped (resampled with replacement) subset of the samples. Further diversity among the trees is generated by basing each tree-split decision in each tree on a different randomly chosen subset of the variables. After the entire forest of slightly different decision trees has been built, it can be applied to new, unseen data by running each new sample down each tree. Just as in CART, each tree's ultimate classification or regression decision is determined by class voting on sample class or the median regression value of the training samples in the case of continuous variables. The aggregate forest's output is then determined by averaging the regression values of the trees or using a weighted voting process to determine the most common class decision reached by the trees. The power of random forests is derived from both the low-bias and the low-variability they achieve on the basis of the 'ensemble' of low-bias, highvariance decision trees.

At the simplest level, the Random Forest algorithm has only two tunable parameters: mTry, the fraction of all variables tried in each tree-split decision, and nTree, the number of trees grown. Typically in Random Forests, nTree is set to 500, but we used nTree $=400$ since that choice showed no appreciable decline in the algorithm's accuracy and achieved a modest increase in efficiency. The best values of mTry, suggested by the literature [14], are $n_{s} / 3$ for regression on a signature vector with continuous values and $\sqrt{ } n_{s}$ if the signature data contains class information, where $n_{s}$ is the number of experimental samples. We used those values, so there were no parameters that we tuned. The algorithm is therefore simple to deploy, and over-parameterization is relatively rare. The Random Forest algorithm also has two other properties that make it especially apt for use within LeFE. The first is that it includes an internal cross-validation procedure that estimates the forest's predictive performance without the need for explicit a priori separation of the testing and training samples. That feature is particularly important in this application because microarray experiments are often run on limited numbers of samples. Because each tree is constructed on a bootstrapped sample representing $1-\mathrm{e}^{-1}$, or approximately two-thirds of the samples, about one-third of the samples are not used to build any given tree. Those unused 'out-of-bag' (OOB) samples are unseen in training and therefore can be used to determine the predictive performance of the tree. After the forest is built, each sample serves as a test case for the approximately one-third of the trees for which it was OOB. That procedure provides an estimate of the forest's error in the prediction for each individual sample. The OOB error of each sample is averaged over all samples to estimate the total error of the model. Fivefold cross-validation and the internal performance assessment using OOB samples have been shown to yield quite similar results [14].

The second useful property of random forests is that they can determine the importance placed on each variable in the model. Each variable's importance is assessed by randomizing the variable's association (permuting the variable's row elements) with the samples and then reassessing the model's error by OOB cross-validation. The Random Forest software package, which we used for the computations, has one iteration as the default, and the documentation states that more than one randomization does not appreciably improve the 


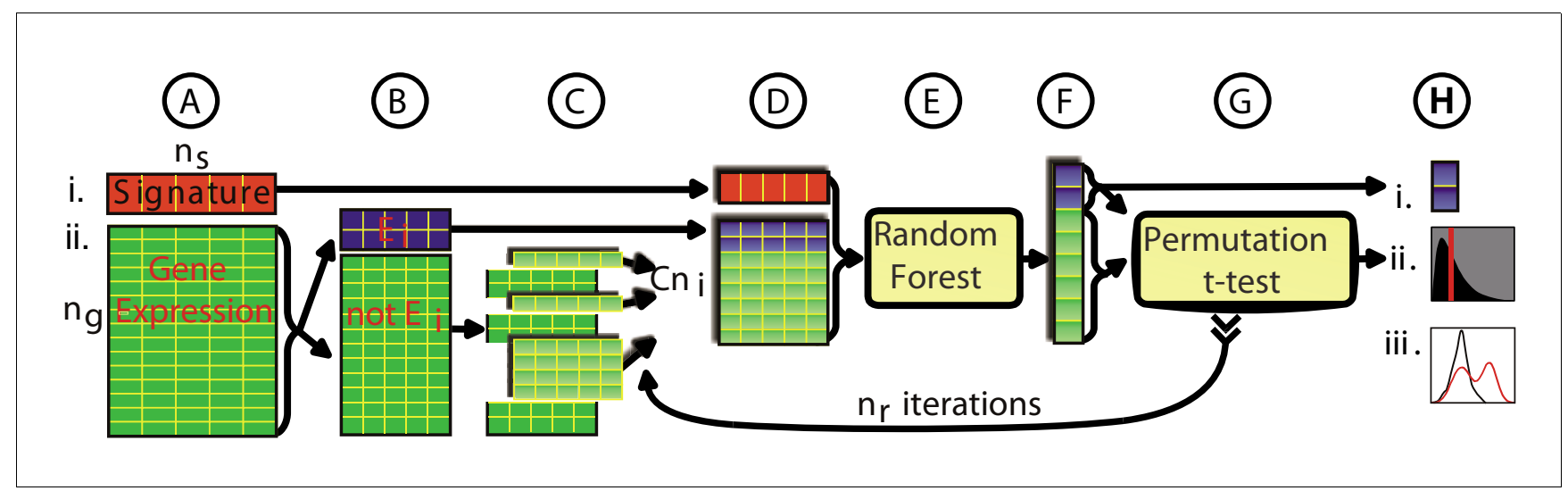

Figure I

The LeFE algorithm illustrated schematically for a category of two genes. See Materials and methods for further details and Table 4 for a description of the steps (keyed to the circled letters). LeFE, Learner of Functional Enrichment.

stability of the calculated importance scores. The loss of model accuracy is normalized by the accuracy of the unpermuted, intact model's performance to give an 'importance score' for each gene in a category. When Random Forest is applied to a classification problem, the model's error is a weighted classification accuracy, and in the regression context model error is the mean squared error. The greater the decrease in normalized performance, the more instrumental was the variable (gene) in achieving the forest's predictive performance. See Materials and methods (below) for a detailed description of the importance score.

The steps in the LeFE algorithm (shown schematically in Figure 1) are described more formally in the Materials and methods section (below). Here, we summarize the basic elements conceptually. For each category, LeFE builds a random forest to model the signature vector on the basis of a composite matrix consisting of genes in the category and a proportionately sized set of randomly selected negative control genes that are not in the category. On that basis, the random forest determines the importance score of each gene (variable) in the multivariate model. The distribution of importance scores of the genes in the category is then compared with the distribution of importance scores of the negative control genes. The expectation is that the two distributions will be similar when that comparison is made for a category that is biologically unrelated to the signature vector. However, if the category includes biologically relevant genes or gene combinations, then Random Forest is expected to assign higher importance scores to at least some of the genes. A onesided permutation $t$-test [19] is used heuristically to compare the distribution of importance scores of the genes in the category with those of the negative control genes. Because the test compares the calculated $t$-scores with the distribution of such $t$-scores obtained after permuting the sample labels (instead of comparing them with a parametric $t$-distribution), it is nonparametric. To ensure diversity in the sampling of negative control genes, that process is repeated $n_{r}$ times, each with the same gene category and a different set of randomly selected negative control genes. As $n_{r}$ becomes large, the random gene sets asymptotically reflect the overall covariance of the dataset. The median of the permutation $t$-test's $P$ values from the $n_{r}$ iterations is taken as an index of the degree of association between the gene category and the signature vector. After LeFE has been applied to each gene category, the categories are ranked according to those median $P$ values.

LeFE is different from the other category-based algorithms listed previously $[2,3,5-7]$ in that it assesses gene importance within the context of a multivariate model. That enables LeFE to access the gene information contained in complex biological interrelationships, rather than relying on the summation of univariate relationships within a category. For example, if two genes in a category were related to the samples' biological process or state by an 'exclusive OR' association, then LeFE could capture that relationship, whereas category-based summations of univariate associations would be likely to overlook it.

\section{Results}

As proofs of principle we applied LeFE to three different prediction problems that represent diverse biological and computational scenarios. The first, current versus never-smoker classification, involvesIdentification of the molecular features that distinguish 57 current smokers from never-smokers on the basis of gene expression profiles of their lung epithelia [20]. The second problem, breast cancer classification, involves identification of characteristic molecular features that classify 49 primary breast cancer microarray samples as basal (estrogen receptor [ER] negative/androgen receptor [AR] negative), luminal (ER positive/AR positive), or 'molecular apocrine' (ER negative/AR positive) [21]. In the third problem, sensitivity to gefitinib, gene expression profiles are used to predict the gefitinib (Iressa, AstraZeneca, London, England) sensitivity of 26 non-small cell lung cancer cell 
Table I

Top 20 LeFE Categories for current versus never-smokers classification

\begin{tabular}{|c|c|c|}
\hline Rank & Category & FDR \\
\hline I & Electron transporter activity BioCarta & $\sim 0$ \\
\hline I & Carbohydrate metabolism (GO:0005975) & $\sim 0$ \\
\hline 1 & Electron transport BioCarta & $\sim 0$ \\
\hline 1 & Glutathione metabolism GenMAPP & $\sim 0$ \\
\hline I & Pentose Phosphate Pathway BLACK & $\sim 0$ \\
\hline 6 & Xenobiotic metabolism (GO:0006805) & 0.016 \\
\hline 6 & O Glycans biosynthesis GenMAPP & 0.016 \\
\hline 8 & PentosePathway BLACK & 0.045 \\
\hline 9 & Protein amino acid O-linked glycosylation (GO:0006493) & 0.069 \\
\hline 10 & Pentose phosphate pathway GenMAPP & 0.094 \\
\hline 10 & Gamma hexachlorocyclohexane degradation GenMAPP & 0.094 \\
\hline 12 & Tyrosine metabolism GenMAPP & 0.097 \\
\hline 13 & Cysteine metabolism (GO:0006534) & 0.105 \\
\hline 13 & GIpPathway BLACK & 0.105 \\
\hline 13 & T cell differentiation (GO:00302I7) & 0.105 \\
\hline 13 & Fatty acid metabolism BioCarta & 0.105 \\
\hline 17 & Retrograde vesicle-mediated transport, Golgi to ER (GO:0006890) & 0.11 \\
\hline 18 & Aldehyde metabolism (GO:000608I) & 0.11 \\
\hline 19 & Digestion (GO:0007586) & 0.114 \\
\hline 19 & MAP0005I Fructose and mannose metabolism GenMAPP & 0.114 \\
\hline
\end{tabular}

FDR, false discovery rate; GO, Gene Ontology; LeFE, Learner of Functional Enrichment.

lines. The continuous-valued signature vector consists of 26 $\log _{10}$ values of the $50 \%$ inhibitory concentrations [22].

\section{Gene categories}

For use in all three applications, we assembled a set of 1,918 nonexclusive gene categories from multiple sources as follows. First, 1,396 gene categories were selected from the GO Consortium's biological process hierarchy. To ensure high quality of the categories, we removed those with evidence codes that denote lower quality assignments: inferred from electronic annotation, nontraceable author statement, no biological data available, and not recorded. Second, 522 gene categories, defined by the MSigDB v1 [2] collection of functional gene sets, was selected. Those categories had been assembled from various sources including BioCarta, GenMAPP, the Human Protein Reference Database, the Human Cancer Genome Anatomy Project, and a large number of manually curated publications.

For the analyses, we mapped the microarray gene annotations to categories and then included all categories in the broad size range from 2 to 150 genes. Because all of the studies used Affymetrix HG-U133A microarrays (Affymetrix Inc, Santa Clara, CA, USA), the mapping process was the same for all three datasets. That filtering process reduced the original set of 1,918 categories to a set of 1,282 . Summaries of the 20 top-ranked categories for all three demonstration applications are given in Tables 1 to 3 . Complete results for the three prediction problems, namely current versus never-smokers classification, breast cancer classification, and sensitivity to gefitinib, are available as Additional data files 1, 2, and 3, respectively.

\section{Current versus never-smoker classification}

Figure 2 shows what we term 'importance plots', which show the distribution of normalized importance scores of genes with respect to their prediction of the signature vector. The red and black curves represent the category's genes and the negative control genes, respectively. Each category is represented by a smoothed distribution, rather than a single value, because the curve represents importance scores calculated for all genes in all $n_{r}$ iterations of the Random Forest algorithm. The glutathione metabolism and aldehyde metabolism categories (positive examples) ranked among the top 20 categories, whereas the viral life cycle category (negative example) ranked 742th out of 1,282. Each of the two positive examples includes at least two peaks: one that corresponds to a peak in the negative control gene distribution (gray arrows) and one or more (red arrows) that reflect the biologically relevant genes. For example, the two top genes in aldehyde metabolism (aldo-keto reductase 1B10 and aldehyde dehydrogenase 3A1) have median importance scores in the peak denoted with 
Table 2

Top 20 categories for breast cancer classification

\begin{tabular}{|c|c|c|}
\hline Rank & Category & FDR \\
\hline I & Breast_cancer_estrogen_signalling GEArray & 0.02 \\
\hline I & Drug_resistance_and_metabolism BioCarta & 0.02 \\
\hline I & FRASOR_ER_UP Frasor_et_al_2004 & 0.02 \\
\hline 4 & mta3Pathway BioCarta & 0.041 \\
\hline 5 & Fatty_Acid_Synthesis BioCarta & 0.065 \\
\hline 6 & Cell_cycle_checkpoint II & 0.065 \\
\hline 6 & p35alzheimersPathway BioCarta & 0.065 \\
\hline 6 & FRASOR_ER_DOWN Frasor_et_al_2004 & 0.065 \\
\hline 9 & L-phenylalanine catabolism & 0.068 \\
\hline 9 & UDP-glucose metabolism & 0.068 \\
\hline 9 & Cell_cycle_regulator & 0.068 \\
\hline 12 & Electron_transporter_activity BioCarta & 0.078 \\
\hline 13 & skp2e2fPathway BioCarta & 0.1 \\
\hline 14 & Fatty_acid_metabolism BioCarta & 0.1 \\
\hline 15 & Ubiquinone biosynthesis & 0.102 \\
\hline 16 & MAP00010_Glycolysis_Gluconeogenesis GenMAPP & 0.12 \\
\hline 16 & MAPKKK_cascade GO & 0.12 \\
\hline 18 & GIPathway BioCarta & 0.134 \\
\hline 18 & MAP00280_Valine_leucine_and_isoleucine_degradation GenMAPP & 0.134 \\
\hline 20 & Response to metal ion & 0.144 \\
\hline
\end{tabular}

FDR, false discovery rate; GO, Gene Ontology; LeFE, Learner of Functional Enrichment.

Table 3

Top 20 categories for sensitivity to gefitinib

\begin{tabular}{|c|c|c|}
\hline Rank & Category & FDR \\
\hline 1 & Androgen up genes na & 0.347 \\
\hline 2 & EGF receptor signaling pathway BioCarta & 0.408 \\
\hline 3 & MAP00I00 Sterol biosynthesis GenMAPP & 0.531 \\
\hline 4 & Epidermal growth factor receptor signaling pathway (GO:0007I73) & 0.531 \\
\hline 5 & $\mathrm{G}_{\mathrm{I}} / \mathrm{S}$ transition of mitotic cell cycle (GO:0000082) & 0.628 \\
\hline 6 & positive regulation of I-kappaB kinase/NF-kappaB cascade (GO:0043I23) & 0.628 \\
\hline 7 & Cell-cell adhesion (GO:0016337) & 0.748 \\
\hline 8 & Aspartate catabolism (GO:0006533) & 0.915 \\
\hline 9 & Calcium-independent cell-cell adhesion (GO:0016338) & 0.915 \\
\hline 10 & Regulation of glycolysis (GO:0006 I I0) & 0.915 \\
\hline 11 & Detection of pest, pathogen or parasite (GO:0009596) & 0.915 \\
\hline 12 & MalatePathway BLACK & 0.915 \\
\hline 12 & RarPathway BLACK & 0.915 \\
\hline 14 & Epidermis development (GO:0008544) & 0.931 \\
\hline 14 & Regulation of endocytosis (GO:0030100) & 0.931 \\
\hline 16 & NFKB reduced Hinata et al 2003 & 0.998 \\
\hline 17 & mRNA editing (GO:000638I) & $\sim 1$ \\
\hline 17 & EMT DOWN Jechlinger et al 2003 & $\sim 1$ \\
\hline 19 & Chloride transport (GO:000682I) & $\sim 1$ \\
\hline 19 & Induction of apoptosis by intracellular signals (GO:0008629) & $\sim 1$ \\
\hline
\end{tabular}

FDR, false discovery rate; GO, Gene Ontology; LeFE, Learner of Functional Enrichment. 


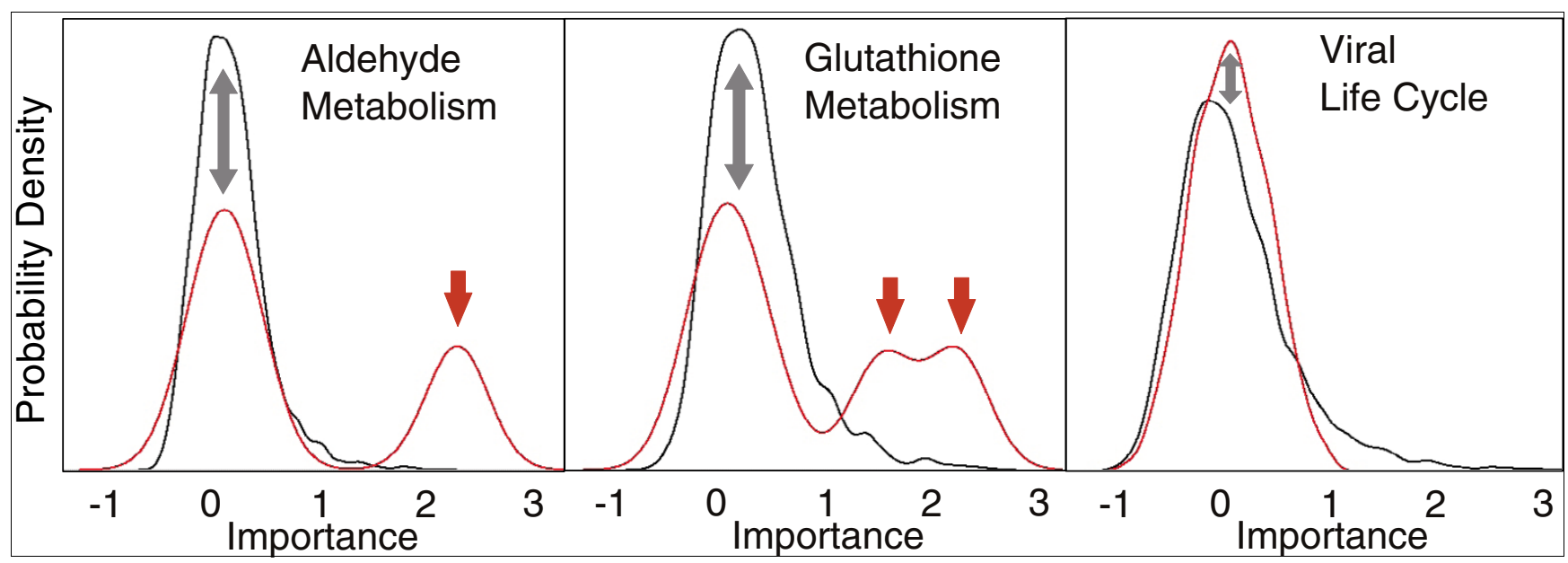

Figure 2

Importance plots (probability density distributions) of gene importance scores calculated by LeFE: smoker versus nonsmoker dataset. Shown are representative distributions for three gene categories (red curves) and their corresponding negative control gene sets (black curves). The curves were smoothed according to default settings of the 'density' function in R. The shifted secondary peaks, denoted by red arrows, for aldehyde metabolism and glutathione metabolism reflect genes important to the Random Forest models. The viral life cycle category contains no secondary peaks and therefore does not appear to be associated with smoking. See Results for further details.

a red arrow, and, as discussed below, they are known to metabolize cigarette smoke toxins [23]. The genes in the viral life cycle category are unrelated to smoking and have distributions indistinguishable from those of the negative control genes.

The highest-scoring five out of the 1,282 categories run through LeFE have median $P<0.001$ (false discovery rate [FDR] < 0.02), and all of them contain genes that are known to exhibit altered expression in response to cigarette smoke in vivo or in vitro. Among the most important genes in the topranked category, electron transport, are $C Y P_{1} B 1, C Y P_{2} A 13$, and $M A O B$, all of which are known to be upregulated by cigarette smoke $[24,25]$. The top genes in the next category, electron transporter activity, include the aldo-keto reductases $A K R 1 B 1 O, A K R 1 C 1, A K R 1 C_{2}$, and $A K R 1 C_{3}$, as well as those encoding aldehyde dehydrogenase $\left(A L D H_{3} A 1\right)$ and monoamine oxidase $\mathrm{B}(M A O B)$. In vivo studies have shown that those genes are upregulated in response to cigarette smoke condensate [23]. The third category, glutathione metabolism, fits with current understanding because glutathione, a tripeptide thiol antioxidant, forms conjugates with cigarette smoke toxins [26]. The fourth ranked category, pentose phosphate pathway, makes sense because, in response to blood plasma previously exposed to cigarette smoke in vitro, endothelial cells have been shown to release glutathione and activate the pentose shunt [27].

Among the top genes in the sixth ranked category, xenobiotic metabolism (FDR = 0.02), are $A K R 1 C 1, C Y P 35 A$, and NQO1. All three have independently been found to be differentially expressed in the bronchial epithelium of smokers [28,29].
Also in the same category is the gene that encodes UDP glucuronosyltransferase 1A6 (UGT1A6). Eight out of the 11 probes for that gene perfectly match the related UGT1A7 gene, which has been shown to detoxify multiple tobacco carcinogens [30]. Hence, the importance score for UGT1A6 may reflect a family resemblance in function, a cross-hybridization of probes, or both.

The 1oth ranked category, $\gamma$-hexachlorocyclohexane degradation $(\mathrm{FDR}=0.09)$, contains several cytochrome $\mathrm{P} 450$ genes with polymorphisms that are known to alter lung cancer risk for smokers. Furthermore, one of that category's highest scoring genes, $C Y P_{1} A 1$, is expressed in primary lung cancer samples in a manner highly correlated with tobacco dose [31]. The 12th ranked category, tyrosine metabolism $(\mathrm{FDR}=0.10)$, contains two previously mentioned aldehyde metabolism genes, $A L D H_{3} A 1$ and $M A O B$. The 16th ranked category, cysteine metabolism $(\mathrm{FDR}=0.11)$, contains only two genes, namely GCLC and GCLM. Together they form the glutamatecysteine ligase complex, which is responsible for increasing the antioxidant glutathione in the lungs of smokers [32].

We next compared LeFE directly with the popular and useful GSEA method [2]. The online documentation of that method suggests that GSEA should not be applied to categories smaller than 25 genes because such categories may produce inflated scores. Abiding by that limitation, GSEA would not have considered 13 of LeFE's top 20 categories, because they include fewer than 25 genes. However, for the sake of this comparison, we chose to ignore the 25-gene limitation and operate GSEA on all categories with a size of at least two. That resulted in a substantial overlap in the top 20 categories iden- 
tified by LeFE and GSEA. However, several categories (including pentose phosphate pathway, aldehyde metabolism, $\gamma$-hexachlorocyclohexane degradation, and cysteine metabolism) that were ranked in the top 20 by LeFE were not in the top 140 categories as ranked by GSEA's FDR, despite the fact that they are all likely to be biologically related to cigarette smoke (see above and Figure 2). Furthermore, LeFE identified 44 categories with FDR below 0.2 and 150 categories with FDR below 0.5, whereas GSEA identified only 18 and 65 , respectively. We cannot state definitively that LeFE did 'better' than GSEA at distinguishing the biology between the two sample classes, but the results do suggest that LeFE's unique method provides a different (although overlapping) set of categories that make considerable biological sense.

\section{Breast cancer classification}

A dominant molecular characteristic of the breast cancer samples is ER- $\alpha$ (ESR1) status. Accordingly, the top categories identified by LeFE are intimately associated with that molecule and related subsystems. Three categories had median $P$ values below $0.001($ FDR $=\sim 0)$ : breast cancer estrogen signaling; MSigDB's set of ER-upregulated genes identified by Frasor and coworkers [33]; and drug resistance and metabolism, which contains ESR1, BCL2, $A R$ and ER's co-regulator $E R B B 4$ [34]. The fourth category, the BioCartadefined MTA3 pathway, contains ESR1 and three estrogenregulated genes, namely $P D Z K 1, G R E B 1$, and $H S P B 1$ (HSP27) [35] as the four most important genes.

Categories related to fatty acid synthesis and metabolism are represented three times in the top 25 categories, with FDRs below 0.02. That result is consistent with the observation that carcinomas of the colon, prostate, ovary, breast, and endometrium all express high levels of fatty acid synthase [36]. Manual literature searches failed to identify independently confirmatory research. However, we analyzed three independent breast cancer studies [37-39] on the Oncomine website [40] using conventional $t$-statistics and confirmed that many of the fatty acid related genes are, indeed, significantly differentially expressed among the three classes of breast cancers. Specifically, PRKAB1, PRKAG1, $P E C I, C R O T, F A B P 7$, and $A C A D S B$ levels were significantly higher in the ER-positive luminal class, whereas PRKAA1 levels were significantly higher in ER-negative samples. FASN, $F A A H$, and $S C N$ were significantly lower in the AR-negative basal samples. The original publications on the datasets analyzed with LeFE noted the altered expression of metabolism genes but failed to identify that fatty acid metabolism systems are associated with breast cancer or breast cancer subtypes. The three categories related to fatty acid synthesis and metabolism contain various combinations of the aforementioned genes and interact with each other in complex ways that distinguish the breast cancer classes. GSEA does not handle multiclass analyses, at least directly, but even if it did it might well have overlooked the fatty acid categories because it depends on univariate associations between genes and sample class.

The three independent breast cancer datasets [37-39] from Oncomine also confirmed our findings for several other categories that had received top LeFE ranks and FDRs below 0.01. L-phenylalanine catabolism, cell cycle regulator, electron transporter activity, skp2e2f pathway, MAPKKK cascade, and response to metal ion (Table 2) contain many genes that received high LeFE importance scores in our LeFE analysis and were also significantly differentially expressed in those independent studies. Precise interpretation of the association between breast cancer and our independently verified genes, which include GSTZ1, BCL2, MPHOSPH6, SRPK1, MCM5, BTG2, SKP2, DUSP7, NRTN, MTL5, NDRG1, and MT1X, is beyond the scope of the present study. A direct comparison of results from GSEA [2] and LeFE for the breast cancer study was not possible because there were three classes.

\section{Gefitinib sensitivity}

Gefitinib inhibits the tyrosine kinase activity of the epidermal growth factor receptor (EGFR) [41]. Accordingly, the second and fourth ranked out of 1,282 LeFE categories are the EGF receptor signaling pathway $(\mathrm{FDR}=0.41)$ and EGFR signaling pathway (FDR $=0.53$ ). If one is accustomed to a critical point such as 0.05 for $P$ values, then an FDR of 0.53 may seem high. However, the implication is that almost half of the time such a category would constitute a true positive, rather than a false positive, even after correction for multiple hypothesis testing. Whether that level of certainty is high enough to act on depends, of course, on the relative cost and benefit of following up the finding. The predictions are clearly not as strong in the case of gefitinib as in the other two applications of LeFE presented here, but some of the top-ranked categories do make biological sense.

The first ranked category, androgen upregulated genes (FDR $=0.35$ ), is interesting because there is evidence that androgen levels increase in non-small-cell lung cancer patients treated with gefitinib [42]. The third-ranked category, sterol biosynthesis (FDR $=0.53$ ), assigns a high importance score to the gene that encodes 3-hydroxy-3-methyl-glutaryl coenzyme A (HMGCR). Gefitinib is synergistic with lovastatin [43], which inhibits HMGCR and is in clinical trials with simvastin, another HMGCR inhibitor, for treatment of non-small-cell lung cancer. That observation suggests the possibility of a link between the sterol biosynthesis pathway and gefitinib's activity.

The association between gefitinib and the fifth-ranked category, $\mathrm{G}_{1} / \mathrm{S}$ transition in mitotic cell cycle (FDR $=0.63$ ), is not completely clear, but it has been shown that EGFR inactivity is required for $\mathrm{G}_{1} / \mathrm{S}$ transition in Drosophila [44]. The seventh category, cell-cell adhesion (FDR $=0.75)$, contains EGFR and Annexin A9, the latter being a cousin of the EGFR substrate Annexin A1. That could represent a novel finding or 
be due to cross-hybridization of the microarray's probes. A comparison of LeFE and GSEA was not possible because GSEA [2] does not operate directly on continuous valued signature vectors.

\section{Discussion}

LeFE is a novel statistical/machine learning method for functional analysis of microarray (and analogous) data. Here, we have implemented it using the Random Forest algorithm with internal cross-validation. LeFE's attention to gene categories differentiates it from earlier microarray analysis methods based on individual genes (for instance, correlation analysis or $t$-tests). Its ability to model complex relationships among the genes within a category also differentiates it from previous category-based ((hyphen necessary to meaning))algorithms (for example, GSEA and methods based on the Fisher's exact test) that are founded on summation of the univariate effects of individual genes within a category. Needless to say, the ability to build more complex models carries with it a potential cost, namely that of 'over-fitting'. However, LeFE's use of negative control gene sets and internal crossvalidation mitigate that concern considerably, and the three proof-of-principle applications described in the Results section speak for themselves. We would not claim that LeFE is 'better' than previous useful methods such as GSEA, but it does clearly have independent value, and it does directly handle problem types (multi-class, continuous valued signature, small categories) that are not handled directly by the other methods.

Our application of LeFE to gene expression in the lung epithelia of current smokers, as opposed to never-smokers, demonstrated its ability to identify and elucidate molecular differences between two sample classes. LeFE correctly identified categories containing the glutathione related genes, aldehyde dehydrogenases, monoamine oxidase, several aldoketo reductases, and cytochrome $\mathrm{P} 450$ genes, all of which are differentially expressed in response to cigarette smoke or in the lungs of smokers. Four of the top biologically important categories were overlooked by GSEA, thereby highlighting LeFE's independent value.

However, a cautionary consideration is in order. Given the vast searchable archives of published biological research, it seemed possible that identifying literature citations consistent with LeFE's findings had a high a priori probability or that it was tainted by multiple hypothesis-testing. To address those possibilities, we designed a simple blinded experiment to test how well LeFE performed in the eyes of a pulmonology expert, Dr Avrum Spira, lead researcher on the lung epithelium gene expression study and first author of the resulting article [20]. We presented him with the top 20 gene categories identified by LeFE, each of them matched with a randomly chosen category of identical, or essentially identical, size. Because some categories have vague names, we also pro- vided the names of the five most important genes in each category. We then asked Dr Spira, who was blinded to the LeFE results, to identify which category in each pair was more likely to be associated with gene expression differences in the epithelium of smokers as opposed to nonsmokers. He correctly distinguished the top seven categories and 17 of the top 20 from their size matched, randomly chosen counterparts. The binary probability of achieving at least 17 out of 20 correct by chance is $P<0.0002$. An additional, independent application of LeFE to the same dataset yielded an overlap of 17 out of the top 20 categories. All three of the new results were correctly identified by Dr Spira.

Our additional applications of LeFE, to gene expression in three breast cancer classes and to in vitro gefitinib sensitivity (see Results), provide further proofs of principle. The findings highlight the distinctions between LeFE and the univariate category based methods. They also underscore the utility of LeFE's novel 'importance plots' for relating the individual gene importance scores to complex relationships within a category.

LeFE's hybrid machine learning/statistical algorithm compares gene categories with sets of randomly selected negative control genes. That approach distinguishes LeFE from the superficially similar PathwayRF [45] program, which was recently reported during the preparation of this paper. The PathwayRF algorithm trains a single random forest on each gene category's genes and then ranks the categories according to the model's predictive accuracy. Unlike LeFE, PathwayRF does not use gene importance scores at all. Results presented in the PathwayRF report indicate that it can provide biologically meaningful insight into gene microarray datasets, but the algorithm has a hidden bias that favors large categories. The predictive power of a statistical or machine learning model increases as independent variables are added if no penalty is imposed for adding those variables, and PathwayRF does not impose such a penalty. Therefore, as shown in Figure 3a, it strongly favors large gene categories because they contain more variables (genes). The mean and median numbers of genes in the top 20 categories for PathwayRF are 68 and 36, respectively. The corresponding values for LeFE are 32 and 22.

PathwayRF's bias toward larger categories can be demonstrated most concretely, as shown in Figure 3 b, by considering the frequently occurring superset-subset (nested) relationships between gene categories in the hierarchically organized GO. With PathwayRF, the superset of a nested category's model is essentially guaranteed to exhibit predictive power at least as great as that of any nested subcategory; all models that can be generated by the subset can also be generated by the superset. (The few points above the diagonal line for PathwayRF in Figure $3 \mathrm{~b}$ are probably there by chance because the algorithm is stochastic in nature.) However, as shown in Figure 3c, even when there is no nesting, larger and 
more biologically diffuse categories are much more likely to do better than smaller, more specific ones. Methods that favor a general hypothesis over a more specific one are likely to mis-prioritize follow-up studies. Therefore, any method in the spirit of LeFE or PathwayRF must correct for category size, and LeFE does that by using a set of negative control genes proportional in size to that of the category.

\section{Conclusion}

In conclusion, we have presented LeFE, a novel statistical/ machine learning algorithm for interpretation of microarray (and analogous) data. LeFE exploits information related to the complex, interactive regulation of gene expression and does not suffer from bias toward large category size. We have demonstrated LeFE's value on three diverse datasets and have shown that the results are either consistent with independently determined biological conclusions or generate novel, plausible hypotheses. A comparison of results from LeFE and GSEA suggests that LeFE identifies important biological information overlooked by the latter method, which does not take into account the complex interrelationships among genes within a category. A new type of visualization, the 'importance plot', captures the distribution of importance scores within a category. Unlike GSEA [2], LeFE is directly applicable to problems with multiple classes or continuously valued signature vectors. A user-friendly program package, LeFEminer, is freely accessible on the internet [46].

\section{Materials and methods Technical description of LeFE Input}

Figure 1 shows a schematic flow diagram of the LeFE algorithm. The first input(indicated by i in Figure 1) is a vector $Y$ of $n_{s}$ sample signature values, each representing a behavior, phenotype, or state of the sample. The signature values may denote classes of samples (for example, for the three breast cancer categories) or continuously distributed values (for example, drug sensitivity). The second input (denoted ii in Figure 1) is a matrix $X$ of gene expression values for $n_{g}$ genes measured over the $n_{s}$ samples. The third input (not shown in Figure 1) is a set $E$ of $m$ gene categories $\left\{E_{1}, E_{2} \ldots E_{i} \ldots E_{m}\right\}$. Each category $E_{i}$ contains $n_{i}$ genes predetermined to be functionally related. Categories can, for example, be GO categories [47] or Kyoto Encyclopedia of Genes and Genomes pathways [1].

\section{LeFE algorithm}

The LeFE algorithm assigns a score that indicates the category's predicted biological association with $Y$. The steps in the algorithm, as applied to a single category, are listed in Table 4, which is keyed to the circled letters in Figure 1.

\section{Output}

The results (not shown in Figure 1) of applying the algorithm to all categories are as follows: a sorted vector of length $m$, representing the ranked median permutation $P$ values of the $m$ gene categories; an importance score for each gene in the context of each category in which it occurs; and an importance plot (provided only for top categories), which shows the distribution of importance scores for all genes in all $n_{r}$ iterations (Figure 2).

\section{Estimation of statistical significance}

The FDR associated with each gene category's median permutation $t$-test value is estimated by permuting the signature vector and calculating the fraction of more extreme scores for data that contain no true biological information. For each of the example analyses described in this report, we have computed FDRs using the method described by Benjamini and Hochberg using 50 independent signature vector permutations [48].

\section{Importance scores}

Gene importance scores were described in general terms in the Introduction (above). A more formal description, adapted from Breiman and Cutler [49], is provided here. For each (microarray) sample $i$ in our experiment, let $\mathrm{X}_{i}$ represent the vector composed of gene expression values of the category's genes and its randomly selected negative control gene set. Let $y_{i}$ represent the sample's true classification or regression value, let $V_{j}\left(\mathrm{X}_{i}\right)$ be the vote of tree $j$ when trained on the values contained in $\mathrm{X}_{i}$, and let $t_{i j}$ be an indicator variable equal to 1 if $i$ is an OOB sample for tree $j$ and otherwise o. Let $\mathrm{X}^{(A, j)}=\left(\mathrm{X}_{1}(A\right.$, $\left.j), \ldots, \mathrm{X}_{N}^{(A, j)}\right)$ represent the gene expression values with the value of gene $A$ randomly permuted among the OOB observations for tree $j$. Then, $\mathrm{X}^{(A)}$ is the collection of $\mathrm{X}^{(A, j)}$ for all trees, where $N$ samples have been selected with replacement from the study's set of $n_{s}$ experimental samples. This notation can easily be used to define importance scores in both the classification and regression contexts if we define the function $f(\alpha, \beta)$. In the context of classification, $f=1$ if $\alpha$ is logically equal to $\beta$ and is otherwise $o$. In the context of regression, $f$ is the mean squared difference between $\alpha$ and $\beta$. Thus, the importance score, $I_{T}$, of variable $A$ is defined as follows:

$$
I_{T}(A)=\frac{1}{T} \sum_{j=1}^{T} \frac{1}{N_{j}} \sum_{i=1}^{N}\left[f\left(V_{j}\left(\mathbf{X}_{i}\right), y_{i}\right)-f\left(V_{j}\left(\mathbf{X}_{i}^{(A, j)}\right), y_{i}\right)\right] t_{i j}
$$

where $T$ is the total number of trees in the forest and $N_{j}$ represent the number of OOB samples for the $j$ th tree. It is then straightforward to see that if the variable $A$ is unimportant and therefore infrequently used, $f\left(V_{j} X_{i}, y_{i}\right) \approx \mathrm{f}\left(V_{j} X_{i}^{(A, j)}\right)$ and $I_{T}(A) \approx 0$.

Importance plots show the distribution of importance scores normalized by the standard error of the inter-tree variances of 


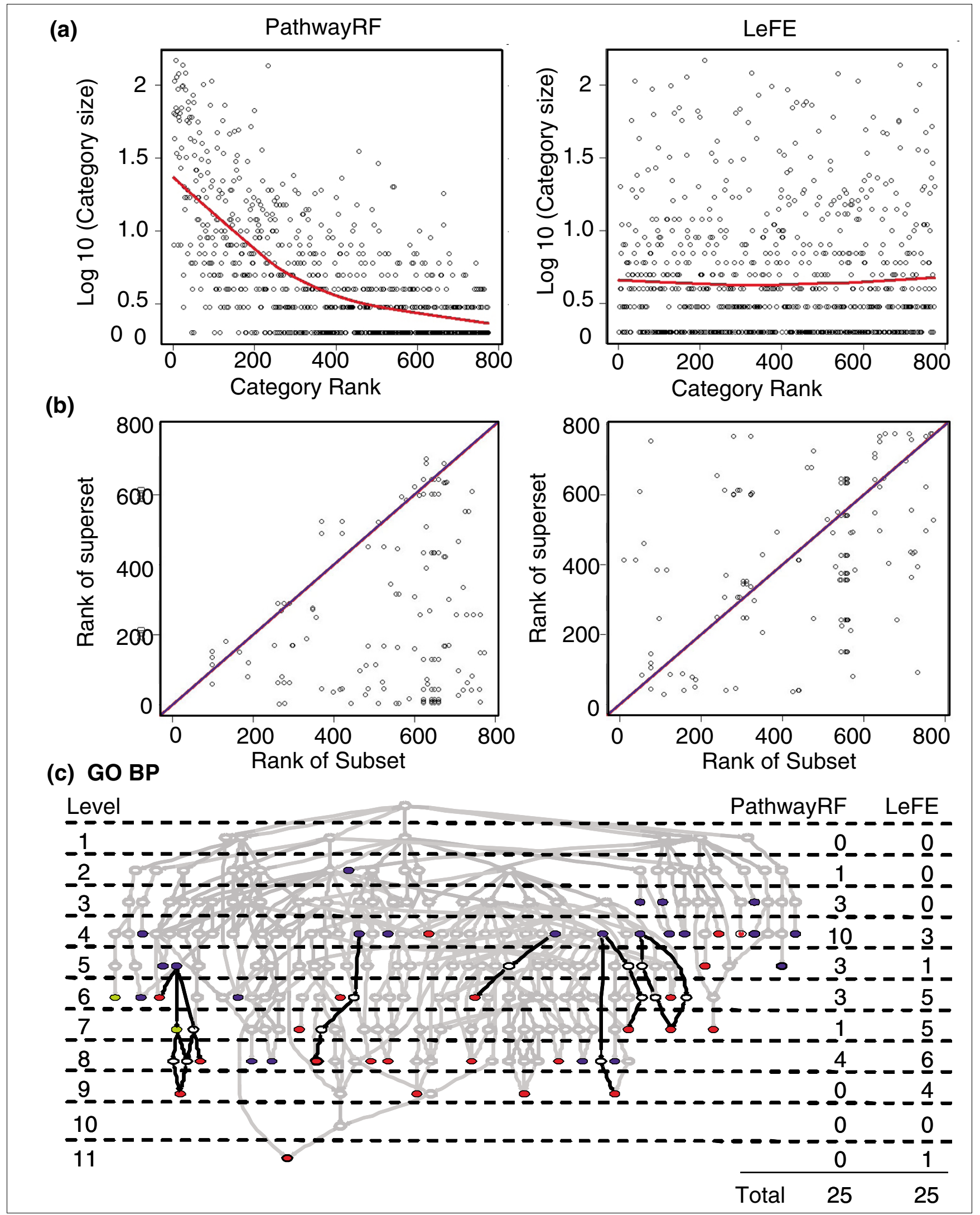

Figure 3 (see legend on next page) 
Figure 3 (see previous page)

A Comparison of LeFE with PathwayRF Shown is a comparison of Learner of Functional Enrichment (LeFE) and PathwayRF with respect to the size distribution of categories identified as important for breast cancer classification using the Gene Ontology (GO) biological process categories. (a) Scatter plots showing category rank versus category size. Ties in category ranks were resolved through random reordering. Red lines are lowess regressions. (b) Comparison of GO superset and subset ranks. Almost all points for PathwayRF are below the blue $x=y$ line, indicating that supersets rank lower (better) than that their corresponding subsets. The panel for LeFE shows no such bias. (c) The GO biological process hierarchy (with the most general categories toward the top). Blue circles denote the top 25 categories ranked by PathwayRF; red circles denote the same for LeFE; and yellow circles denote categories in the top 25 for both algorithms. The mean GO level is 4.92 for PathwayRF and 7.08 for LeFE. There are no cases in which LeFE's top results are the ancestors of top results from PathwayRF. However, the black edges highlight eight cases in which LeFE found categories that are progeny of categories identified by PathwayRF.

$I_{T}(\mathrm{~A}), \sigma_{T}^{2}$. Therefore, the normalized importance scores are as follows:

$$
Z_{T}(A)=\frac{I_{T}(A)}{\sqrt{\sigma_{T}^{2}(A) / T}}
$$

The importance plot shows two smoothed probability density distributions of importance scores: one for the genes in the category (red curve) and the other for the sets of negative control genes (black curve). If category $i$ has $\left|E_{i}\right|$ genes, then the red distribution (of the category's genes) is composed of $n_{r} \times$ $\left|E_{i}\right|$ genes, and the black distribution (with corresponding negative control genes) has $n_{r} \times\left|E_{i}\right| \times C$ genes, where $C$ is an integer constant defined in Table 4 (step C).
Importance scores are usually positive values, but it is possible for them to take on negative values if the model's performance happens to improve when the variable is permuted.

Because importance scores are computed within the model's context, they reflect the complex, multivariate relationships among genes in a category, even though they are assigned to individual genes. That duality is key to the LeFE algorithm.

\section{Implementation of LeFE}

We implemented the algorithm in R v2.4 [50], using Bioconductor [51], and the randomForest R package. Multiprocessor capabilities in the Rmpi and snow $\mathrm{R}$ libraries were used to speed up LeFE's extensive computations. A user-friendly, Java-based web application, LeFEminer, is freely available on

Table 4

\section{Steps in the LeFE algorithm}

Step Details

A The gene expression matrix, signature vector, and gene categories (not shown in Figure I) are entered

B For each category $E_{i}$, the genes in the microarray $(X)$ are split into those in $E_{i}$ and those not in $E_{i}$ (denoted, respectively, by two blue and $I 2$ green rows of the gene expression matrix)

C A negative control set consisting of $C \times n_{i}$ genes in $X$ but not in $E_{i}$ is selected at random. Those genes are noted as elevated rows in Figure $I$, which was arbitrarily drawn for $C=3$. The integer constant $C$ is used to mitigate issues of statistical imprecision associated with small categories. The default value $C=6$ creates better run-to-run reproducibility and was used in the actual LeFE calculations

D A composite matrix of gene expression $X_{i t e r}$ is assembled by selecting the rows in $X$ that correspond to the $n_{i}$ genes in $E_{i}$ along with the negative control genes selected in step $C$. The resulting data structure, $X_{i t e r}$, has dimension $\left(C \times n_{i}\right)+n_{i}$ rows by $n_{s}$ columns

E A random forest with 400 trees is built on $X_{i t e r}$ to model the signature vector, using the default value for the random forest parameter $m$ Try. The random forest is given no information for distinguishing between category genes and negative control genes

$\mathrm{F} \quad$ A vector $l$ of standardized importance scores of each gene in $X_{\text {iter }}$ is computed internally from the random forest. $l$ is then divided into two sets of importance scores, $I_{E}$ and $I_{\text {not }}$, for the genes in $E_{i}$ and the negative control set, respectively

G The statistical significance of the gene expression evidence for rejection of the null hypothesis that the mean of $I_{E}$ is less than or equal to the mean of $I_{\text {notE }}$ (that the category genes are, on average, more important to the Random Forest model than the negative control genes) is determined by a one-sided permutation $t$-test. Because that test compares observed $t$-statistics with a null distribution of $t$-statistics of permuted data, it avoids using the parameterized $t$-distribution and is therefore nonparametric. For statistical robustness, steps $C$ to $G$ are repeated $n_{r}$ times with different, randomly selected sets of negative control genes. The covariate structures of the $E$ and not $E$ genes are likely to differ, but the negative control genes, selected at random from notE, are unbiased with respect to the ranking of categories in the next step and with respect to the calculation of false discovery rates

$\mathrm{H} \quad$ Applying the above procedure to a single gene category creates three outputs. The first (denoted $i$, in Figure I) is a median importance score for each of the $n_{i}$ genes in the category. Because the median importance scores are computed within the category's multivariate Random Forest models, they reflect the genes' importance within its complex biological context. The second output (denoted ii) is the entire category's median $P$ value from the $n_{r}$ permutation $t$-tests. The third output (denoted iii) is an importance plot, which compares the distributions of importance scores of the genes in the category and the negative control genes

Shown are the steps in the Learner of Functional Enrichment (LeFE) algorithm, with the letters in the left-hand column corresponding to those in Figure I. 
the internet [46]. It enables nontechnical experimentalists and biologists to apply the algorithm to their datasets. To perform LeFE's heavy computational tasks, the web service application is multiprocessed on six $1.5 \mathrm{GHz}$ 64-bit Itanium processors with 4 GB RAM at the National Cancer Institute's Advanced Biomedical Computing Center. Analysis of a typical microarray experiment takes 90 to $240 \mathrm{~min}$.

To manage the high computational requirements of running the LeFE algorithm, and because most users are most interested in the top-ranked gene categories, a slightly modified version of LeFE is supported by the LeFEminer web application [46]. Median permutation $t$-test $P$ values are rapidly estimated by the LeFE algorithm with $n_{r}=10$ iterations instead of the more accurate (and time consuming) $n_{r}=75$ iterations. Based on that preliminary ranking of each category's likelihood of being important, LeFEminer prioritizes the calculation, running up to $n_{r}=75$ iterations for the top categories and fewer for the less promising ones according to a logistic curve. Using the logistic curve, LeFE sets $n_{r}$ to be at least 60 for the top 40 preliminarily ranked categories and then gradually reduces $n_{r}$ until it settles at 20 for the least promising (roughly $85 \%$ ) of the categories. That slight modification preserves the accuracy and reproducibility of the results for the top ranked categories while using only a fraction of the computation time. LeFEminer uses the increased efficiency to estimate the FDR of the results. Replicate applications of the faster version of LeFE to the three datasets analyzed (current versus never-smokers, breast cancer type, and gefitinib sensitivity) produced highly reproducible rankings, with Spearman correlation coefficients (calculated over all of the ranks) ranging from 0.95 to 0.98 (see Figure 4 and Table 5).

\section{Reproducibility of LeFE}

Because LeFE is not a deterministic algorithm, reaching full computational convergence may require relatively high values of $n_{r}$ and $n_{c}$, along with random forests of considerable size. We used $n_{r}=75, n_{c}=6$, and $n$ Tree $=400$, respectively, in the present calculations. Figure 4 shows the reproducibility of LeFE for the breast cancer classification dataset. The overall Spearman correlation over replications was $r=0.99$, and the correlation of the top 50 categories was $r=0.82$. The results of repeated analyses using the more efficient LeFEminer

Table 5

Correlation of ranks between two applications of LeFE with different random number generator seeds

\begin{tabular}{lll}
\hline Dataset & Overall correlation & Top 50 correlations \\
\hline Breast cancer & 0.98 & 0.70 \\
Current Smoker/ & 0.97 & 0.70 \\
Never- Smoker & & \\
Gefitinib & 0.95 & 0.61 \\
\hline
\end{tabular}

LeFE, Learner of Functional Enrichment.

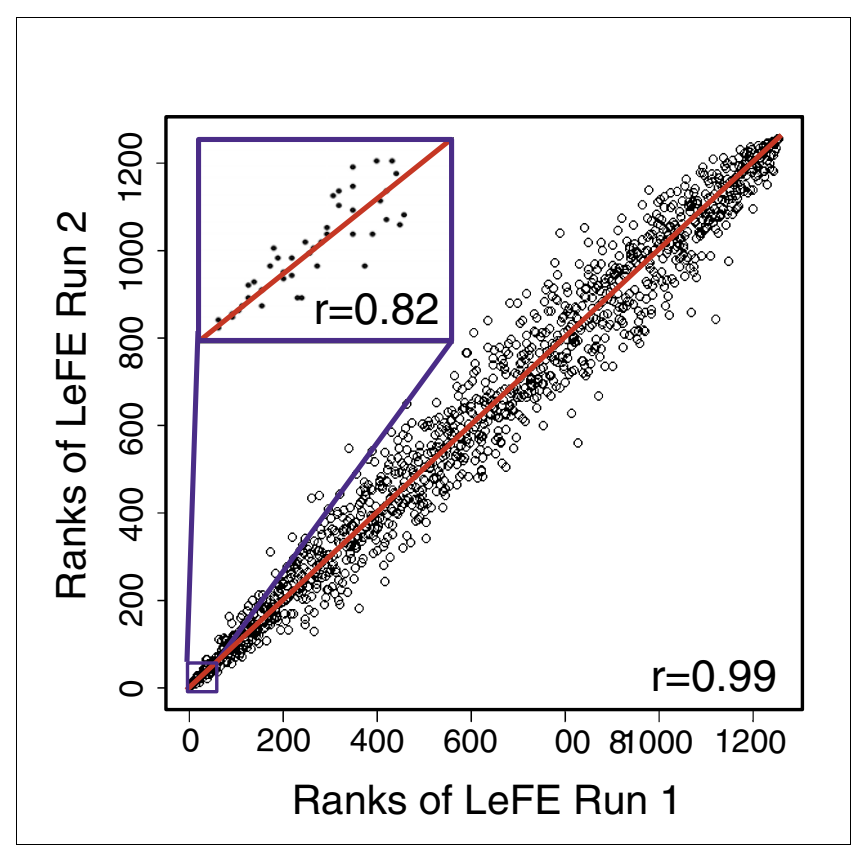

Figure 4

Replicate applications of LeFE to the breast cancer classification dataset. Scatter plot comparing the ranks resulting from two applications of Learner of Functional Enrichment (LeFE) to the breast cancer classification dataset, with $n_{r}=75, n_{c}=6$, and $n$ Tree $=400$. The inset represents a blowup of the top 50 categories. $r$ denotes the Pearson's correlation coefficient of the ranks (the Spearman correlation coefficient).

implementation (see above) on all three datasets are summarized in Table 5 .

\section{Data for use in LeFE}

LeFE and its internal Random Forest algorithm can be operated on a variety of prediction problem types. Unlike GSEA [2], it directly handles regression and multi-class classification. However, its flexibility creates a limitation worth discussion. LeFE's Random Forest algorithm requires a collection of samples that exemplify diverse states or behaviors. The default setting of the randomForest $\mathrm{R}$ package used by LeFE grows each decision tree until the tree's leaves have no fewer than five samples in a regression model or one sample in a classification model. Because the goal of LeFE is to capture any gene interactions, the simplest logical models not captured by standard univariate methods must contain at least two genes. Despite the observation that random forests consider only a subsampling of variables to make each decision tree split, LeFE can, in fact, be applied to small categories with as few as two variables (genes). That is so because the random forests are actually constructed on composite categories of at least $2 n_{c}+2=14$ genes in the case of a category with two genes and $n_{c}=6$. As an informal test of whether LeFE was doing something reasonable with small gene categories, we examined the highest ranked two-gene categories ( $\mathrm{T}$ cell differentiation and cysteine metabolism) in the current-smoker/ never-smoker dataset. In each case, the low permutation 
t-test $P$ values were explained by the observation that both genes in the category had high importance scores.

\section{Abbreviations}

$\mathrm{AR}$, androgen receptor; CART, Classification and Regression Trees; EGFR, epidermal growth factor receptor; ER, estrogen receptor; FDR, false discovery rate; GO, Gene Ontology; GSEA, Gene Set Enrichment Analysis; LeFE, Learner of Functional Enrichment; OOB, out-of-bag; RF, Random Forest.

\section{Authors' contributions}

GE formulated the original concept of LeFE and implemented the R scripts; he also ran all of the demonstration calculations, built the website's entire back-end and parts of the front-end, and wrote the most of the manuscript. MR consulted on statistical aspects of the LeFE algorithm. DK built the web application's front-end and middle-tier communications layers. JNW consulted on the algorithm and biological results and also helped to write the manuscript.

\section{Additional data files}

The following additional data are available with the online version of this paper. Additional data file 1 provides results from the current/never-smoker demonstration. Additional data file 2 provides complete results from breast cancer demonstration. Additional data file 3 provides complete results from the gefitinib demonstration.

\section{Acknowledgements}

We thank Dr. Avrum Spira for his independent validation of our results for the lung epithelium dataset and Philip Lorenzi, Barry Zeeberg, Uma Shankavaram, and Bill Reinhold in the Genomics \& Bioinformatics Group for insightful discussion and suggestions. We also thank the National Cancer Institute's Advanced Biomedical Computing Center for providing and supporting computational resources used in this work. This research was funded by the National Cancer Institute's Center for Cancer Research.

\section{References}

I. Kanehisa M: A database for post-genome analysis. Trends Genet 1997, 13:375-376.

2. Subramanian A, Tamayo P, Mootha VK, Mukherjee S, Ebert BL, Gillette MA, Paulovich A, Pomeroy SL, Golub TR, Lander ES, et al.: Gene set enrichment analysis: a knowledge-based approach for interpreting genome-wide expression profiles. Proc Natl Acad Sci USA 2005, I02: $15545-15550$.

3. Zeeberg BR, Feng W, Wang G, Wang MD, Fojo AT, Sunshine M, Narasimhan S, Kane DW, Reinhold WC, Lababidi S, et al:: GoMiner: a resource for biological interpretation of genomic and proteomic data. Genome Biol 2003, 4:R28.

4. Zeeberg BR, Qin H, Narasimhan S, Sunshine M, Cao H, Kane DW, Reimers M, Stephens RM, Bryant D, Burt SK, et al:: High-Throughput GoMiner, an 'industrial-strength' integrative gene ontology tool for interpretation of multiple-microarray experiments, with application to studies of common variable immune deficiency (CVID). BMC Bioinformatics 2005, 6:168.

5. Khatri P, Draghici S, Ostermeier GC, Krawetz SA: Profiling gene expression using onto-express. Genomics 2002, 79:266-270.
6. Martin D, Brun C, Remy E, Mouren P, Thieffry D, Jacq B: GOToolBox: functional analysis of gene datasets based on Gene Ontology. Genome Biol 2004, 5:RIOI.

7. Castillo-Davis $\mathrm{Cl}$, Hartl DL: GeneMerge: post-genomic analysis, data mining, and hypothesis testing. Bioinformatics 2003, 19:89|-892.

8. Michel JB, Ordway GA, Richardson JA, Williams RS: Biphasic induction of immediate early gene expression accompanies activity-dependent angiogenesis and myofiber remodeling of rabbit skeletal muscle. J Clin Invest 1994, 94:277-285.

9. Pugh BF: Control of gene expression through regulation of the TATA-binding protein. Gene 2000, 255: $|-| 14$.

10. Ayoubi TA, Van De Ven WJ: Regulation of gene expression by alternative promoters. FASEB J 1996, 10:453-460.

II. Lohr D, Venkov $P$, Zlatanova J: Transcriptional regulation in the yeast GAL gene family: a complex genetic network. FASEB J 1995, 9:777-787.

12. Breiman L: Random Forests. Machine Learning 200I, 45:5-32.

13. Blower PE, Cross KP, Eichler GS, Myatt GJ, Weinstein JN, Yang C: Comparison of methods for sequential screening of large compound sets. Comb Chem High Throughput Screen 2006, 9:115-122.

14. Svetnik V, Liaw A, Tong C, Culberson JC, Sheridan RP, Feuston BP: Random forest: a classification and regression tool for compound classification and QSAR modeling. J Chem Inf Comput Sci 2003, 43: 1947-1958.

15. Lunetta KL, Hayward LB, Segal J, Van Eerdewegh P: Screening large-scale association study data: exploiting interactions using random forests. BMC Genet 2004, 5:2.

16. Shi T, Seligson D, Belldegrun AS, Palotie A, Horvath S: Tumor classification by tissue microarray profiling: random forest clustering applied to renal cell carcinoma. Mod Pathol 2005, 18:547-557.

17. Institute of Electrical and Electronics Engineers, IEEE Neural Networks Council: IEEE Transactions on Evolutionary Computation: A Publication of the IEEE Neural Networks Council New York, NY: Institute of Electrical and Electronics Engineers; 1997.

18. Breiman L: Classification and Regression Trees Belmont, CA: Wadsworth International Group; 1984.

19. Simon RM: Design and Analysis of DNA Microarray Investigations New York, NY: Springer; 2003.

20. Spira A, Beane J, Shah V, Liu G, Schembri F, Yang X, Palma J, Brody JS: Effects of cigarette smoke on the human airway epithelial cell transcriptome. Proc Natl Acad Sci USA 2004, 1 01 : I 01 43-10 148.

21. Farmer $P$, Bonnefoi $H$, Becette $V$, Tubiana-Hulin M, Fumoleau P, Larsimont D, Macgrogan G, Bergh J, Cameron D, Goldstein D, et al.: Identification of molecular apocrine breast tumours by microarray analysis. Oncogene 2005, 24:4660-467I.

22. Coldren CD, Helfrich BA, Witta SE, Sugita M, Lapadat R, Zeng $C$, Baron A, Franklin WA, Hirsch FR, Geraci MW, et al.: Baseline gene expression predicts sensitivity to gefitinib in non-small cell lung cancer cell lines. Mol Cancer Res 2006, 4:52I-528.

23. Nagaraj NS, Beckers S, Mensah JK, Waigel S, Vigneswaran N, Zacharias W: Cigarette smoke condensate induces cytochromes $\mathbf{P 4 5 0}$ and aldo-keto reductases in oral cancer cells. Toxicol Lett 2006, 165:182-194.

24. Port JL, Yamaguchi K, Du B, De Lorenzo M, Chang M, Heerdt PM, Kopelovich L, Marcus CB, Altorki NK, Subbaramaiah K, et al:: Tobacco smoke induces CYPIBI in the aerodigestive tract. Carcinogenesis 2004, 25:2275-228I.

25. Su T, Bao Z, Zhang QY, Smith TJ, Hong JY, Ding X: Human cytochrome P450 CYP2A 13: predominant expression in the respiratory tract and its high efficiency metabolic activation of a tobacco-specific carcinogen, 4-(methylnitrosamino)-I-(3pyridyl)-I-butanone. Cancer Res 2000, 60:5074-5079.

26. Rahman I, MacNee W: Lung glutathione and oxidative stress: implications in cigarette smoke-induced airway disease. Am J Physiol 1999, 277:L1067-1088.

27. Noronha-Dutra AA, Epperlein MM, Woolf N: Effect of cigarette smoking on cultured human endothelial cells. Cardiovasc Res 1993, 27:774-778.

28. Wiemels J, Wiencke JK, Varykoni A, Smith MT: Modulation of the toxicity and macromolecular binding of benzene metabolites by NAD(P)H:Quinone oxidoreductase in transfected HL-60 cells. Chem Res Toxicol 1999, I 2:467-475.

29. Woenckhaus M, Klein-Hitpass L, Grepmeier U, Merk J, Pfeifer M, Wild P, Bettstetter M, Wuensch P, Blaszyk H, Hartmann A, et al.: Smoking and cancer-related gene expression in bronchial 
epithelium and non-small-cell lung cancers. I Pathol 2006, 21 0:192-204.

30. Zheng Z, Park JY, Guillemette C, Schantz SP, Lazarus P: Tobacco carcinogen-detoxifying enzyme UGT IA7 and its association with orolaryngeal cancer risk. J Natl Cancer Inst 200I, 93:14|I-1418.

31. Anttila S, Tuominen P, Hirvonen A, Nurminen M, Karjalainen A, Hankinson O, Elovaara E: CYPIAI levels in lung tissue of tobacco smokers and polymorphisms of CYPIAI and aromatic hydrocarbon receptor. Pharmacogenetics 200I, I I:50I-509.

32. Neurohr C, Lenz AG, Ding I, Leuchte H, Kolbe T, Behr J: Glutamate-cysteine ligase modulatory subunit in BAL alveolar macrophages of healthy smokers. Eur Respir J 2003, 22:82-87.

33. Frasor J, Danes JM, Komm B, Chang KC, Lyttle CR, Katzenellenbogen BS: Profiling of estrogen up- and down-regulated gene expression in human breast cancer cells: insights into gene networks and pathways underlying estrogenic control of proliferation and cell phenotype. Endocrinology 2003, 144:4562-4574.

34. Zhu Y, Sullivan LL, Nair SS, Williams CC, Pandey AK, Marrero L, Vadlamudi RK, Jones FE: Coregulation of estrogen receptor by ERBB4/HER4 establishes a growth-promoting autocrine signal in breast tumor cells. Cancer Res 2006, 66:799|-7998.

35. Ghosh MG, Thompson DA, Weigel RJ: PDZKI and GREBI are estrogen-regulated genes expressed in hormone-responsive breast cancer. Cancer Res 2000, 60:6367-6375.

36. Kuhajda FP, Pizer ES, Li JN, Mani NS, Frehywot GL, Townsend CA: Synthesis and antitumor activity of an inhibitor of fatty acid synthase. Proc Natl Acad Sci USA 2000, 97:3450-3454.

37. Sotiriou C, Neo SY, McShane LM, Korn EL, Long PM, Jazaeri A, Martiat P, Fox SB, Harris AL, Liu ET: Breast cancer classification and prognosis based on gene expression profiles from a population-based study. Proc Natl Acad Sci USA 2003, 100:10393-10398.

38. van de Vijver MJ, He YD, van't Veer LJ, Dai H, Hart AA, Voskuil DW, Schreiber G], Peterse JL, Roberts C, Marton MJ, et al.: A geneexpression signature as a predictor of survival in breast cancer. N Engl J Med 2002, 347:1999-2009.

39. Wang Y, Klijn JG, Zhang Y, Sieuwerts AM, Look MP, Yang F, Talantov $D$, Timmermans M, Meijer-van Gelder ME, Yu J, et al.: Gene-expression profiles to predict distant metastasis of lymph-nodenegative primary breast cancer. Lancet 2005, 365:67|-679.

40. Rhodes DR, Yu J, Shanker K, Deshpande N, Varambally R, Ghosh D, Barrette T, Pandey A, Chinnaiyan AM: ONCOMINE: a cancer microarray database and integrated data-mining platform. Neoplasia 2004, 6: I-6.

4I. Ono M, Hirata A, Kometani T, Miyagawa M, Ueda S, Kinoshita H, Fujii T, Kuwano M: Sensitivity to gefitinib (Iressa, ZD I 839) in nonsmall cell lung cancer cell lines correlates with dependence on the epidermal growth factor (EGF) receptor/extracellular signal-regulated kinase I/2 and EGF receptor/Akt pathway for proliferation. Mol Cancer Ther 2004, 3:465-472.

42. Nishio M, Ohyanagi F, Horiike A, Ishikawa $Y$, Satoh $Y$, Okumura S, Nakagawa K, Nishio K, Horai T: Gefitinib treatment affects androgen levels in non-small-cell lung cancer patients. $\mathrm{Br} J$ Cancer 2005, 92:1877-1880.

43. Dimitroulakos J, Lorimer IA, Goss G: Strategies to enhance epidermal growth factor inhibition: targeting the mevalonate pathway. Clin Cancer Res 2006, I 2:4426s-443 Is.

44. Baker NE, Yu SY: The EGF receptor defines domains of cell cycle progression and survival to regulate cell number in the developing Drosophila eye. Cell 200I, 104:699-708.

45. Pang H, Lin A, Holford M, Enerson BE, Lu B, Lawton MP, Floyd E, Zhao H: Pathway analysis using random forests classification and regression. Bioinformatics 2006, 22:2028-2036.

46. LeFEminer [http://discover.nci.nih.gov/lefe]

47. Ashburner M, Ball CA, Blake JA, Botstein D, Butler H, Cherry JM, Davis AP, Dolinski K, Dwight SS, Eppig JT, et al:: Gene ontology: tool for the unification of biology. The Gene Ontology Consortium. Nat Genet 2000, 25:25-29.

48. Benjamini $Y$, Hochberg $Y$ : Controlling the false discovery rate: a practical and powerful approach to multiple testing. J Roy Stat Soc 1995, 57:289-300.

49. Breiman L, Cutler A: Random Forests. Version 5. [http:// www.stat.berkeley.edu/ breiman/RandomForests/cc_home.htm].

50. The R Project for Statistical Computing [http://www.rproject.org]
5I. Bioconductor: Open Source Software for Bioinformatics [http://www.bioconductor.org] 